\title{
29. COMMISSION DES SPECTRES STELLAIRES
}

Président: M. O. Struve, Yerkes Observatory, Williams Bay, Wisconsin, U.S.A.

Membres: MM. W. S. Adams, E. A. Baker, Barbier, Beals, Beer, Cecchini, Cesco, Chalonge, Colacevich, Dunham, Edwards, Erro, Fehrenbach, Mme Payne-Gaposchkin, MM. Gratton, Greaves, Gregory, Herzberg, Hiltner, F. S. Hogg, Hynek, Joy, Keenan, Kharadze, Lacroute, O. J. Lee, Lindblad, Mme Davis Locanthi, MM. McKellar, McLaughlin, Mme Walton Mayall, MM. N. U. Mayall, Melnikov, P. W. Merrill, Milne, Minkowski, W. W. Morgan, Nassau, Petrie, Redman, Russell, Sahade, Sanford, G. A. Shajn, Stratton, Swings, Thackeray, Vorontsov-Velyaminov, Vyssotsky, Weaver, R. C. Williams, O. C. Wilson.

Président: M. Stratton.

\section{Sous-Commission des Novae}

Membres: MM. Baade, C. Bertaud, Cecchini, Mme Payne-Gaposchkin, MM. Gratton, McLaughlin, P. W. Merrill, Minkowski, Struve, Swings, Zwicky.

Sous-Commission des Bandes Moléculatres dans les Spectres Stellaires

PrÉsident: M. McKellar.

Membres: MM. Adel, Fehrenbach, Herzberg, Keenan, Sanford, Swings.

\section{THE FUNDAMENTAL PROBLEMS}

The Commission on Stellar Spectra records great progress in its field of investigation. We have reached the stage where a concerted attack can be made on two problems of fundamental importance which consciously or unconsciously have been the principal aim of stellar spectroscopists. The first is the problem of the abundances of the chemical elements in their relation to the question of nuclear transformations and the origin of the elements. The other is the problem of the origin of the stars and of their subsequent evolution.

Our knowledge of the abundances of the elements in the solar reversing layer, originally due to Russell, has been further increased through the work of Wildt, Chandrasekhar, and others who have solved the problem of the continuous absorption coefficient; of B. Strömgren, who applied the apparatus of modern physics to the construction of theoretical line-profiles and determined from them the important ratio of hydrogen to metal abundances; and of Chalonge and Barbier, as well as Chandrasekhar and Münch, who made use of the absorption coefficient of $\mathrm{H}^{-}$to explain the energy distributions of the continuous spectra of all but the hotter stars.

The determination of abundances in the stars seemed almost within our grasp 20 years ago when Russell and Adams published their classical paper on the spectra of several bright stars observed with the Mount Wilson Coudé spectrograph. The discovery in I934 of the peculiar features of the stellar 'curves of growth', and especially of the unexpected phenomenon of turbulence, retarded progress, so that at the Stockholm meeting neither Commission 29 nor Commission 36 (Spectrophotometry) felt inclined to discuss this problem. The intervening ten years have seen a gratifying change. The work of Unsöld on $\tau$ Sco gave us the abundances in a normal early B star and demonstrated the unexpectedly high values for He and Ne. Unsöld's recent work on the abundances in the solar reversing layer shows an amazing similarity to $\tau$ Sco (and to the meteorites) despite the fact that $\tau$ Sco is located within the great obscuring clouds of Ophiuchus, and that it belongs to the Scorpius-Centaurus cluster. Aller made important progress in the study of curves of growth of $\mathrm{O}, \mathrm{B}$, and A stars; K. O. Wright found remarkable differences in 
the curves of growth of several $F$ stars, which depend upon excitation potential (especially for $\mathrm{Fe}$ I) and upon stage of ionization; and Struve suggested that the turbulent velocity may increase with the height in the atmospheres of some F-type supergiants. There is a strong suggestion, especially in some unpublished work of Schwarzschild and perhaps also in that of $\mathrm{K}$. O. Wright, that in these supergiants the turbulent elements are not small as compared with the extent of the layer, and also that the distribution of velocities may not be Maxwellian. The most recent advance is that of Greenstein who found that normal $\mathrm{F}$ stars, including members of the main sequence, giants, and supergiants, have remarkably similar abundances (within less than a factor of two). But a 'metallic-line' A star can only be explained by assuming a vastly different distribution of some of the elements.

While the painstaking analysis of individual line profiles and the elucidation of the features of the curves of growth are required for detailed studies, much progress can be made by relatively crude methods. Popper's and Bidelman's discoveries of faint stars of early type without $\mathrm{H}$ lines are reminiscent of the earlier work at Mount Wilson and Yerkes on $v$ Sgr and at Lick on R CrB. Such cases can be explained, at present, only by assuming an abnormally low abundance of $\mathbf{H}$. Not many years ago our only suspicion of differences in abundance came from the $\mathrm{C}$ and $\mathrm{N}$ differentiation of the $\mathrm{W}$ stars.

It is tempting to seek in the cases of small hydrogen abundance a manifestation of the process of nuclear transformation of $\mathrm{H}$ into He. But at present we cannot draw such a conclusion. Not only is it dangerous to generalize for the entire star results pertaining to only a very thin outer fringe, but we must be prepared to find evidence of other physical processes, such as stratification, rotation, radiation pressure, etc., and of differences in composition resulting from regional differences in the original medium. There are indications of such effects: the low abundance of $\mathrm{H}$ in the nebula surrounding the B-type component of $\alpha$ Sco may be the result of radiation pressure, and the peculiar line intensities of some A-type stars may be related to their magnetic fields ( $\mathrm{H}$. W. Babcock).

Future work will probably be directed along several different lines. There must be further work on the curve of growth in order to dispose of questions relating to the properties of the turbulent motions. The best conclusion now is to regard these motions as arising in something like a vast field of prominences (Menzel, Struve). It is possible that the forces which operate in these prominences are selective (Shajn) and that this may produce an apparent anomaly of chemical composition in the supergiants and shell stars. Even more promising should be studies of individual line-profiles with instruments of higher resolving power than are available to-day. The interpretation of these profiles by the method of Strömgren should greatly reduce the present uncertainty.

The abundances of the isotopes of $C$, among others, are of special interest according to recent work at Victoria by McKellar, at McDonald by Herzberg, and at Saint Michel by Fehrenbach. The causes of these differences are still obscure but McKellar and Fermi have suggested that they may have some connection with the fact that in the Bethe cycle $\mathrm{C}^{13}$ would be used up more rapidly than $\mathrm{C}^{12}$, so that if the original abundance of . $\mathrm{C}^{13}$ was abnormally large the cycle would gradually bring about equilibrium at $\mathrm{C}^{12} / \mathrm{C}^{\mathbf{1 3}} \sim 90$. Progress has been slow because few contemporary stellar spectroscopists are sufficiently acquainted with the theory and even the practice of molecular spectroscopy. The Commission calls attention to this fact and expresses the hope that several astrophysicists in different countries will undertake this field of study. This is especially important because relatively few pure physicists are now working in spectroscopy.

Another promising start could be made with instruments of moderate dispersion to determine whether there are any spectroscopic differences in stars of the same type depending upon differences in origin or physical characteristics. For example, the location of the main sequence in the HR diagram determines the theoretical abundance of $H$. It would be important to compare the spectra of stars of the same type in clusters having different HR diagrams. The work of Trumpler and of others has given us a beginning. With a dispersion of $50 \mathrm{~A} . / \mathrm{mm}$. considerable progress should be possible (this suggestion is in part due to a conversation with Baade and Merrill). The remarkable weakness of 
the absorption lines of the B-type Pleiades, quite aside from their systematically large velocities of rotation, is of interest in this connection. Special attention should also be directed to the pronounced correlation between spectroscopic characteristics of stars belonging to Baade's populations I and II, and their motions.

The problem of regional differences in the spectra of the stars is intimately connected with that of their origin and evolution. In this field the principal advance was made by Joy, who recently announced the discovery of some forty peculiar bright-line stars in and near the dark absorbing clouds of Taurus. This remarkable discovery is the culmination of the long and painstaking process of recording innumerable small features of peculiar bright-line stars principally by Merrill, Sanford, Joy, and others at Mount Wilson, by Swings and Struve at McDonald, by McLaughlin at Michigan, Neubauer at Lick, etc. It is the first clear-cut connection between peculiar spectroscopic features and location in or near a cosmic dust cloud. As Greenstein and Aller have pointed out, only faint, small stars show this connection. Ordinary stars of early and intermediate spectral classes have normal spectra. If the evolution of a real star resembles that described in the theoretical work of Spitzer and Whipple, then we may well wonder whether such an object as the intrinsically faint emission-line star which may be responsible for the relatively large reflection nebula Bro of Taurus may not be an embryonic star, and whether the interaction between the particles of the cloud and the radiation of the star may not produce the emission lines of the latter and (perhaps through a process of fluorescence) the abnormally large surface brightness of the former.

\section{THE PHYSICAL PROPERTIES OF STELLAR ATMOSPHERES}

The most significant advance in this field during the years covered by this report was the discovery in I946, by $\mathrm{H}$. W. Babcock, at Mount Wilson, of magnetic fields up to about I0,000 Gauss, in a number of stars belonging to the peculiar group of strontiumeuropium objects of classes A and F. A sequence to Hale's work on the magnetic field of the sun, this new result opens a large area for observational and theoretical study. It is intimately connected with the problem of stellar rotation and with that of the peculiar sequences among the A stars. The spectra of physical variables have been exhaustively investigated at Mount Wilson. Among the many conclusions of this work we record Merrill's opinion that the $H$ emission lines of long-period variables may originate below the conventional level of the photosphere (because the metallic absorption lines which cut into these emission lines at their first appearance may be stronger than would be inferred from lines which fall in the ordinary continuous spectrum). The spectra of Cepheid variables with high dispersion are under investigation by Adams and Schwarzschild, as well as by Swings and his collaborators. Earlier results by Pannekoek, Walraven and Van Albada in Amsterdam (from Victoria plates) and by Struve at McDonald suggest that some lines may originate in a shell-type layer (partly supported against gravity), and that the line profiles and radial velocities do not necessarily record the true velocity of the deeper pulsating layers. In this connection the simultaneous existence of two displaced $\mathrm{H}$ lines near the phase of median increasing brightness of RR Lyrae is of interest, and the undisplaced emission lines (Struve, Sanford) may be caused by the interaction of the two colliding layers. This process (which has been discussed for nebulae by Oort and Burgers) may be of great importance in stellar spectroscopy.

At Victoria, Petrie has nearly completed the measurement of $\Delta m$ in some Ioo spectroscopic binaries with two spectra. The results are of great value in connection with studies of the mass-luminosity relation. The spectra of peculiar stars, especially those with emission lines, have given a wealth of new information. It is instructive to realize that sometimes the same observational phenomenon-in this case the presence of emission lines-may be produced by entirely different causes - turbulence or collisional processes in relatively deep atmospheric layers of some Cepheids and perhaps of the long-period variables; fluorescence in extended nebulous envelopes of some 'symbiotic stellar spectra' (a term suggested by Merrill for such stars as $Z$ And, AX Per, etc.). The identification 
problem of the emission lines, especially of the forbidden lines, and the interpretation of their intensities has been advanced especially by Swings, Bowen and others. The former suggests that a search be made for forbidden lines in the near infra-red region (e.g. [Fe I] 8347.55).

Studies of peculiar emission-line objects at Mount Wilson and McDonald have already been mentioned. The excitation of emission through fluorescence is fairly well understood, largely through the work of Bowen, Swings, Thackeray, Merrill and others. The study of Be stars and their spectroscopic variations has been continued by McLaughlin at Michigan and by Gregory in London. Heard at Toronto has made a comprehensive study of the peculiar shell-star HD 218393. Merrill is working on the third section of his catalogue of Be stars and he requests observers to send him notes on discoveries and observations of $\mathrm{Be}$ and Ae stars; lists of references would also be welcome. Dufay at Lyon and Miss Hase in the U.S.S.R. have investigated the spectrum of $\gamma$ Cas. Especially impressive is the progress made in the study of W stars, by Swings and Hiltner at McDonald, by O. C. Wilson at Mount Wilson and by Aller at Lick and Indiana. Some of the surprising results of this work are the large percentage of spectroscopic binaries, the systematic displacements of different emission lines, the absence of Wilson's expected time lag between light minimum and velocity curve, and especially Kopal's and Mrs Shapley's conclusion that the emitting shell of a W star is nearly opaque to the radiation of the B-type companion eclipsed by it. The spectra of several P Cygni stars were investigated by Beals at Victoria. A most amazing case of an object with several rapidly changing expanding shells producing several simultaneous violet-displaced absorption components has been the subject of an unpublished investigation by Minkowski.

Studies of other peculiar stars include those of Keenan and Hynek on the perplexing variations of the OI lines in VV Cephei; and of Popper, Bidelman, Greenstein, Thackeray, Keenan, Swings and Larson-Leander on the equally surprising changes in the absorption spectrum of $\rho$ Cas. The eclipsing binaries have led to the discovery of gaseous streams in close systems (Joy, Struve, S. Gaposchkin) and to the conclusion that the bright CaII lines are usually produced in rather small 'tidal bulges' of late-type components of binaries (Joy, Hiltner) which must not be too close to one another (W UMa stars do not have such emission lines). Emission lines of $\mathrm{Ca} I \mathrm{I}$ in $d \mathrm{~K}$ and $d \mathrm{M}$ stars have been the subject of studies by Joy at Mount Wilson, Popper at McDonald, and Gratton at Merate.

Spectra of high dispersion have given a wealth of information on peculiar lineduplicities (Adams); asymmetries (Adams, Greenstein) and identifications (Miss Davis, Swensson, Swings and Struve). Among the most noteworthy advances during the past Io years was the study by Wellmann at Berlin-Babelsberg and especially by $O$. C. Wilson at Mount Wilson of the structure of a giant atmosphere from high-dispersion plates of $\zeta$ Aurigae during its partial eclipses. On Mount Wilson Coudé plates of M-type supergiants Spitzer found interesting differences in the central intensities of strong Fe I lines which depend upon excitation potential and also, in the case of one multiplet, upon a peculiar process of fluorescence. The latter phenomenon is related to that investigated earlier by Thackeray in long-period variables. The central intensities in the absorption lines of early-type stars (Unsöld, Greenstein, Miss Underhill) are also best explained as the result of fluorescence, though there appear to be interesting differences between B-type supergiants and main-sequence stars.

The Stark effect of the He I lines has been the subject of several discussions. Miss Douglas and Foster stressed the peculiar fact that the measured stellar $\lambda$ of the forbidden $(2 \mathrm{P}-4 \mathrm{~F})$ line, $\lambda 4470$, is almost identical with the predicted $\lambda$ for zero field strength at which the theoretical intensity should be nil (this was first noticed by Struve). They proposed a fascinating explanation in terms of a peculiar cycle; observational results by W. Petrie and Miss Underhill at first seemed to confirm the existence of such a cycle. But experience from the study of the He I cycles in shell spectra (Wurm, Struve) would rather tend to throw doubt upon the operation of a powerful cycle in stars of the main sequence where physical factors (such as dilution of radiation) are probably not conducive to the formation and maintenance of the cycle. An important advance was made by 
Mrs Kiess-Krogdahl, who followed up an idea of Chandrasekhar and showed that the theory of microscopic inter-ionic fields in an ionized stellar atmosphere differs markedly from that of a uniform, large-scale field, such as was considered by Foster. The new theory reduces the discrepancy between theory and observation, but perhaps does not completely remove it. The existence of the Foster-Douglas cycle should be further investigated.

Collisional broadening of spectral lines, hardly recognized ten years ago, has become a commonplace phenomenon. Unsöld, Lindholm, Mrs Kiess-Krogdahl, and several others have made theoretical and observational studies of this phenomenon.

Notable progress has been made, especially by Kuiper, in the study of the spectra of white dwarfs, sub-giants and other special groups. The long-neglected group of spectrum variables has been investigated by Deutsch for the determination of the periods and by Swings and Struve and also by Tai, for their physical properties. Ordinary changes of ionization are not sufficient to explain the observed variations of line intensities, and we are again faced with the necessity of attributing unusual abundances to certain elements (for example, to the rare earths).

The work of Chalonge, Barbier and other French investigators on the continuous spectra of the stars, and especially on the Balmer discontinuity, has already been commented upon. The identification problem in absorption spectra, though well advanced, is by no means solved. Swings calls attention to the urgent need of laboratory investigations of some doubly ionized metals, especially Ni III, Cr III, Mn III, Ti III, and of some doubly ionized rare earths, especially Eu III, Gd III, Dy III (under way at Liége). It is probable that the large number of unidentified lines, especially in some peculiar A stars, could be greatly reduced if these spectra were known.

\section{SPECTRAL CLASSIFICATION}

When the I.A.U. was founded this problem was the only one which came within the scope of Commission 29. Since that time the name of the Commission has been changed several times depending upon whether the principal task was to deal with the technical problem of classification or with the physical interpretation of the spectra. The aims of the classifier are often far removed from those of the astrophysicist: he may have little or no concern with the physical properties of the stars and desire information only concerning their luminosities and distances. It may be desirable, now or in the future, to discuss whether a separate commission should be organized to deal with classification problems alone. But while many persons engaged in spectral classification could probably get along quite well without the astrophysical interpretations, the astrophysicist cannot get along without the help of his colleague who is interested in classification. Hence, for the immediate future the Commission believes that the present arrangement should be continued and that later, if there should be a sufficient desire for more detached deliberation of classification problems, a sub-committee be formed for this purpose.

One of the earlier reports of this Commission expressed the hope that a set of reproductions of typical stellar spectra be prepared in order to standardize the Henry Draper system of classification. This has now been accomplished through the publication of the Morgan-Keenan-Kellmann Atlas, of which a new and greatly enlarged edition is in preparation. It should be noted that several members of this Commission have complained that the Atlas is not available at many of even the most important observatories.

Because of Dr Morgan's experience in problems of spectral classification I have asked him to prepare a brief statement concerning some of the outstanding problems:

It does not appear that there will be a marked increase in accuracy in the determination of spectral types in the near future; progress is more likely to take place in the application of methods already developed to general problems of stellar astronomy. Among many promising lines of inquiry the following may be noted especially:

I. The segregation of groups of stars of similar absolute magnitude for investigations of galactic structure. The development of Schmidt objective prism cameras of the type now in 
use at Cleveland and Tonanzintla has made possible large-scale extensions of the spectroscopic parallax methods formerly used on slit spectrograms. It is now possible to identify supergiants of all spectral types to photographic magnitude Io or II on objective prism spectrograms having a dispersion of around $300 \mathrm{~A} . / \mathrm{mm}$. in the blue region. This makes possible additional investigations of certain features of our system at distances of the order of that to the galactic centre. It would also be a simple observational problem to prepare a catalogue of all $B$ stars brighter than the twelfth magnitude and within a few degrees of the galactic equator. The importance of such a catalogue-especially when combined with photoelectric colour determinations-cannot be over-emphasized.

2. A survey for the discovery of stars of special astrophysical interest; for example, the spectrum variables of class $A$ can be recognized by certain spectral peculiarities visible with very low dispersion.

3. When slit spectrograms are used having a dispersion in the photographic region of 60I30 A./mm., it is possible to derive fairly accurate absolute magnitudes and parallaxes for non-peculiar stars of all types. The most difficult to discuss at present are probably stars of classes $\mathrm{B}_{9}-\mathrm{A}_{3}$, while the most accurate absolute magnitudes can be derived for the $\mathrm{Bo}-\mathrm{B}_{3}$ stars and those of classes $\mathrm{G}_{5}$-Ko having absolute magnitudes fainter than zero. It can be stated safely that at present there are hundreds of thousands of stars within the reach of accurate spectroscopic parallax methods; the only things that are needed to bring in this rich harvest are the time to obtain the plates and to make the necessary observations and reductions. A programme having for its object the determination of absolute magnitudes and parallaxes of all stars brighter than the tenth magnitude by objective prism methods is perfectly feasible at present; the whole programme could be completed within a few years if embarked on by a carefully organized group of observers.

Alfred $\mathrm{H}$. Joy of Mount Wilson writes that in order that the Harvard system of stellar classification may be made more accessible to various observers, the time seems opportune to propose a set of standard stars which, after due consideration by experienced observers, might eventually be adopted by the International Astronomical Union. It would be a great advantage if the existing system of classification which has been approved by the Union and is extensively used throughout the world, could be maintained in its essential features without change until such time as a more comprehensive system based on physical parameters can be devised. Dr Joy has, therefore, prepared a list of stars which represent the Harvard system in its simplest form, and he will send a copy to those who request it. He would be glad to have criticisms from the members of the Commission as to its usefulness and suggestions as to the suitability of the individual stars.

A. N. Vyssotsky recommends the adoption of ten standard areas in each of which fifty or more stars between magnitudes 8 and II pg. should be classified as to luminosity and temperature according to W. W. Morgan's criteria. These should be used to establish a sound statistical basis for the system of classification of any instrument or observer dealing with faint stars. The following suggested list of areas may serve as a basis for discussion.

\begin{tabular}{|c|c|c|c|c|}
\hline Designation & R.A. & Decl. & Long. & Lat. \\
\hline S.A. 8 & $1^{\text {h }} 00$ & $+60^{\circ}$ & $92^{\circ}$ & 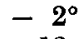 \\
\hline S.A. 22 & 238 & +45 & 111 & -13 \\
\hline S.A. 94 & 252 & +0 & 144 & -4 \\
\hline S.A. 122 & $\begin{array}{ll}6 & 13\end{array}$ & -15 & 190 & -1 \\
\hline S.A. 98 & $\begin{array}{ll}6 & 48\end{array}$ & -0 & 181 & + \\
\hline S.A. 33 & 1350 & +45 & 57 & +67 \\
\hline S.A. 134 & $18 \quad 10$ & -15 & 343 & \\
\hline S.A. 111 & 1935 & +0 & 6 & -12 \\
\hline 22 Cyg. & $19 \quad 52$ & +38 & 41 & + \\
\hline S.A. 41 & 2150 & +45 & 61 & \\
\hline
\end{tabular}

Note that the first and the next to last of these areas are rich in B stars. Furthermore, the selection allows a comparison with the Bergedorf, Potsdam, and H.D.E. systems. 
A communication from A. Schwassmann dated August 7, I938, which had arrived too late for the Stockholm meeting, recommends the preparation of a 'Spektrale Himmelskarte', providing for stellar spectra what the Franklin-Adams charts provide for direct images. A Schmidt camera of $60 \mathrm{~cm}$. aperture and $\mathrm{I} \cdot 2 \mathrm{~m}$. focal length, with a linear dispersion of $2 \mathrm{~mm}$. between $\mathrm{H} \beta$ and $\mathrm{H} \epsilon$ would register spectra of stars to phot. mag. 13.5 in one hour, over a field of 50 square degrees. The Commission will wish to consider this recommendation. Perhaps one of the existing Schmidt telescopes could undertake this task.

Actual progress in the classification of stellar spectra has been most encouraging. Morgan and Bidelman's principal contribution has been the accurate classification of many bright stars, especially those of class B, which are complete to mag. 6.5 and are now being extended to mag. 9.0. The continuation of the HD extensions by Mrs Mayall and their publication are being eagerly awaited. Vyssotsky has completed 37, I0o faint stars in the A.G. zones $-2^{\circ}$ to $+10^{\circ}$; $+10^{\circ}$ to $+20^{\circ}$; and $+30^{\circ}$ to $+50^{\circ}$; and some I0,000 stars of known p.m. He has discovered 270 new $d \mathrm{M}$ stars. Extensive work is in progress at Saltsjöbaden and Uppsala. (A summary of these investigations with bibliography, by Lindblad, appeared in $A p . J$. 104, 325, I946.) Lindblad reports that special attention is being devoted to the study of 'racial' differences among stellar spectra. Ramberg is concerned with the stars in Cepheus and Lacerta, Elvius with those in selected areas, Larsson-Leander with open clusters, Setterberg has completed a study of $\mathrm{CN}$ and $\mathrm{CH}$ in late-type stars, and Brahde one of $\mathrm{T} \mathrm{CrB}$. Zonn of Warsaw has investigated at Saltsjöbaden the spectra of $\delta \mathrm{Cep}, \eta$ Aql and o Cet, while Miss Iwanowska of Torun has made a study of the spectra of high-velocity stars. The Dearborn Observatory has completed its survey of red stars. Classification work at Case School by Nassau, McCuskey and others has only recently been started. The advantages of the 36-inch Schmidt telescope with objective prism are so great that we may well look forward to an unprecedented increase of our knowledge of the spectra of faint stars from this source. Even more recent is the objective prism work inaugurated by Erro, Haro, Rivera and others with the new Tonanzintla (Mexico) Schmidt telescope. The Palomar I8-inch Schmidt was used by Humason and Zwicky for a search of faint blue stars.

We have no direct information concerning the classification work by Schwassmann in Germany. According to Dutch sources Vol. 3 of the Bergedorf Spektraldurchmusterung $\left(\right.$ zone $+30^{\circ}$ ) was ready for publication before the end of the war. This volume was received at the Yerkes Observatory while the report was in the Press. This Commission records its interest in the continuation of the valuable Spektraldurchmusterung.

The classification of $O$ and $B$ stars with a slit-spectrograph of average dispersion by Petrie at Victoria has led him to suggest $(a)$ that the classification by means of estimated line ratios be abandoned, (b) that a sequence of standard stars be established separately for giants and for dwarfs, for rotating and for non-rotating stars, which have been investigated at one or more observatories for line-intensity, and $(c)$ that the classification of other, fainter stars be based upon direct comparisons with these sequences. Swings has remarked that several W stars are now known to possess lines of carbon and nitrogen with similar intensities so that the classification of these objects may have to be revised.

\section{INSTRUMENTS AND OPPORTUNITIES FOR RESEARCH}

In the field of instrumentation we have witnessed an extension into the extreme infrared region by means of lead-sulfide photoconductive cells (Kuiper, Whitford, McMath, Adel, Mohler) and into the region of radio-frequencies ('cosmic static'). The near ultraviolet has been thoroughly explored with aluminized mirrors, gratings and quartz prisms. Rocket spectroscopy has given us a knowledge of the solar spectrum to about $\lambda 2200$. In the ordinary photographic region, which now extends from $\lambda 3000$ to about $\lambda$ 10,000 there has been a tendency to use more powerful spectrographs, and some of the most successful consist entirely of mirror systems with gratings as dispersing units. The enormous increase in the efficiency of diffraction gratings ruled by Wood at Johns Hopkins and by H. D. Babcock at Mount Wilson has created a revolution in spectrograph design. 
Equally important was the invention of fast photographic emulsions in the red and near infra-red regions (especially by the Eastman Co.). Swings has remarked that still further progress may be expected by means of the evaporographic technique. The nearer infra-red has been explored by photographic means, especially at the Perkins Observatory.

The non-reflecting coatings of lenses and prisms have not only increased the efficiency of many spectrographs of more conventional design, but have helped still further to eliminate scattered light-an advantage which in some instances has led observers to prefer good prisms to good gratings. An interesting development was the tendency at Mount Wilson to concentrate an increasing amount of observing at the Coudé focus. Plans are under way, for at least one large telescope of the future, to eliminate altogether the usual type of Cassegrain spectrograph, and to work only at the Coude and the prime foci. Perhaps equally spectacular was the development of fast nebular spectrographssome of them using solid-block Schmidt cameras-especially at the Lick (N. U. Mayall) and Mount Wilson (Humason) Observatories. These instruments quite regularly record the spectra of objects which cannot be seen on the slit, so that for successful operation the guiding must be done on a field star. For purposes of spectral classification several large Schmidt telescopes have been built, the most successful to date being the 36-24-inch instrument at Case Institute of Technology in Cleveland. The completion of the Palomar Mountain 200-inch reflector and 72-48-inch Schmidt telescope will undoubtedly lead to new and startling developments in stellar spectroscopy. Modern electronic methods have had a small, but encouraging amount of application. Thus, it is customary to use electronic amplifiers for the recording of infra-red spectra. An unusual photoelectric guiding device at the Coudé focus of the Mount Wilson roo-inch (H. W. Babcock) has brought realization to a century-old dream of the astronomer: to let a machine do the work of the observer in the dome. Some physicists (Dieke) have expressed the opinion that direct photoelectric recording of spectra has now reached such a stage of perfection that it should be used for many, if not all, astrophysical observations. Attempts in this direction, ten or more years ago, were not too encouraging. But technical developments during the war have been so great that a new effort should be made to eliminate the photographic procedure in the study of line contours and equivalent widths. The difficulty of compensating for 'variable seeing' can probably be overcome by means of differential measurements in two wave-lengths. Hiltner and Code have started such an instrument for high-resolution scanning. Along the more conventional lines the inauguration of spectroscopic work at Córdoba, by Gaviola, Sahade and others was of the utmost importance since it represented the first step toward equalization of effort in the southern and northern hemispheres. But, as some members of this Commission have stressed, there is still a deplorable lack of spectrographic results from the southern regions. In this connection Dunham, in collaboration with the Fund for Astrophysical Research, has prepared a plan for a 70-inch telescope-spectrograph which would operate in the Coudé form and for which some of the mirrors and disks are already in existence. Dunham proposes that this instrument be mounted somewhere in South Africa for the purpose of photographing spectra of southern stars, without interrupting the programmes of other, more universal, telescopes. This Commission records its interest in this project and urges that astronomers interested in the spectra of southern stars communicate with Dr Dunham in order to solve the remaining financial problems-perhaps on a collaborative basis.

On the debit side of this Report we must record the wanton destruction by the German armies of the Simeis reflector with its spectrographic equipment and of the entire equipment at Pulkovo. Perhaps equally disastrous, though understandable as a just retribution, was the dismantling of the Berlin-Babelsberg Observatory after the war, which removed an active institution from research in stellar spectroscopy and which, to date, has not brought about a revival of observational work in stellar spectroscopy in Russia. These, and other losses caused by the war, are far more important than is evident on the surface. They have still further reduced interest in and understanding of stellar spectroscopy among the astronomers of several countries in Europe. This Commission is seriously concerned over this situation. It wishes to bring to the attention of all members of the 
I.A.U. that without a thorough appreciation and understanding of stellar spectroscopy there can be no real progress in many other, apparently unrelated, fields of astronomy, such as the study of variable stars, of proper motions, of visual binaries, etc. The Commission considers it important to combat the defeatist attitude of many astronomers in war-torn countries to abandon efforts in stellar spectroscopy, and in consequence to lose interest in its results, because of the realization that only wealthy nations can afford the large telescopes required for this type of work. In an attempt to further this purpose the Commission recommends:

I. That the I.A.U. adopt a programme for the proposed international observatory that would include equipment for stellar spectroscopy with slit and slitless instruments.

2. That members of Commission 29 make full use of the offer extended to them as the result of previous correspondence from the directors or astronomers of the Mount Wilson, Lick, Victoria, Perkins and McDonald Observatories to secure from these institutions prints (or glass reproductions), microphotometer tracings and other material suitable for original research. Requests for such material (which should be accompanied by a detailed outline of the proposed investigation and should be intended for immediate use) may be addressed to the directors of these observatories, to the spectroscopist members of Commission 29, or to the chairman of this Commission. Although it may not be possible to fill all requests, there is a large amount of material available which can be investigated without having access to the telescopes.

3. That foreign astronomers visiting the large observatories should not necessarily take the view that it is useless for them to spend their limited time in stellar spectroscopy because after their return home they would be unable to continue work in this field, and that consequently they should devote themselves exclusively to work requiring inexpensive instrumental facilities. A reasonable fraction of these visitors should, on the contrary, establish close ties with stellar spectroscopists in those countries where the necessary equipment exists and arrange some sort of co-operative work with them that could last after their return home. In another branch of astronomy and in another era the work of Kapteyn has demonstrated how much may be accomplished in this manner.

4. That stellar spectroscopists in the larger institutions be encouraged to publish material which they have not had the time to discuss. An example of this is the HiltnerWilliams Atlas of tracings from McDonald Observatory Coudé spectrograms. This Atlas contains a wealth of information on curves of growth, abundances of elements, etc., which has not yet been utilized. Stratton's Atlas of Nova Herculis, I934, is another example. Plans are now under way, at Merrill's suggestion, to publish for limited distribution occasional issues, perhaps as an appendix to the Astrophysical Journal and probably by a cheap off-set process, which will contain extensive tabular material and other bulky data not suitable for the regular issues of a journal. This Commission welcomes the plan and recommends that observers make available to others, by its means, tabular and other data which may be only incompletely digested. For example, it would be appropriate to publish in this form a list of equivalent widths in an unusual spectrum like $\alpha \mathrm{CVn}$, or in a Cepheid variable. Authors would hesitate to print incomplete studies in the regular issues of a scientific journal. In some cases the distribution of half-tones or even of photographic reproductions of unusual spectra, such as Novae, shell-stars, spectrum variables like $\rho$ Cas, etc. would furnish material for those who now lack it and would at the same time solve the problem of several large observatories which are now unable to keep up with the discussion of the plate material in their files.

\section{REPORT OF SUB-COMMITTEE ON NOVAE}

In a Symposium held in Paris in 1939 under the presidency of $H$. N. Russell and with the support of the Singer-Polignac Foundation the problems of Novae and Supernovae were fully discussed (see Actualités Scientifiques et Industrielles, Nos. 901, 902, 903; Hermann: Paris, 194I). The present report will confine itself to later publications and to still outstanding problems. 
Novae. Recent observations of earlier Novae have included photographs of Nova Herculis I934 taken at Mount Wilson in red and blue light ten years after the outburst. They show an expanding elliptical shell with condensations at the ends of the major axis. The sight-line velocities of the red and violet maxima have changed but little in ten years, but the expanding shell is now large enough to show that different spectral lines are not equally strong over the whole shell. The lines [O III], 4959, 5007 are strongly present over the shell and in the condensations at the ends of the major axis, but are weak at the ends of the minor axis, where the lines [N II] 6548,6584 are strong.

A recent photograph (r944) taken at Mount Wilson of Nova Persei Igor suggests strongly that the irregular condensations expanding from the Nova are part of an elliptic shell of which for some unexplained reason only a portion is visible.

The later spectrograms of Nova Herculis I934, including such lines as those of [Fe VI], [Fe VII], suggest resemblances to the stars surrounded by shells examined by Struve and Swings $(A p . J .9 \mathbf{9}-8)$. The problem of shells, both stationary and expanding but simultaneously present, is akin to that of successive shells surrounding the central star of a Nova.

Of the Novae which have appeared since I939 the most interesting is $T$ Coronae Borealis, which had an outburst in I 946 , resembling in many details the previous recorded outburst in I866. On this second occasion we have had the unusual opportunity of watching spectral changes in the star for some years preceding the outburst. The rapid fading, without changes of velocity, of a high-velocity absorption spectrum caught at the Yerkes Observatory just after the outburst suggests the blowing out and dispersal of an existing shell already surrounding the star.

Another interesting object was Nova Cygni 1942, a slow Nova which in its spectral changes at the deep drop in brightness closely followed Nova Herculis I934. (For a possible explanation of the phenomena accompanying the deep minima in slow novae see ter Haar, M.N.R.A.S. 104, 283, 1947.)

For a comparative study of a number of Novae reference may be made to Bertaud (Ann. de l'Obs. de Paris, Section d'Astrophysique à Meudon, 9, I, I945) and Cecchini and Gratton (Publ. d. R. Assoc. Astr. de Milano, I939), but above all to a series of papers on the general problems of Novae by McLaughlin (in particular Publ. Obs. University of Michigan, 8, No. I2, I942). He has discussed the relations existing between the time, the absolute magnitudes at maximum, the light curves, spectral sequences, and the velocity of outflow of atoms and also the resemblances and differences between fast and slow Novae.

In this necessarily very brief summary of the fresh material of the last few years much has been omitted. Two points remain to be added: (I) a list of further observational data urgently required; and (2) a list of problems for discussion at the meeting in I 948.

(I) (a) Infra-red spectra of Novae at different stages, especially to aid in a search for molecular bands. Infra-red spectra of Nova Puppis I 942 were secured at the McDonald Observatory, but only for the immediate post-maximum stage.

(b) Further spectrophotometric study of (i) the continuous spectrum, with an attempt to separate when possible the radiation from the central star and that from a surrounding envelope; (ii) the changing relative intensity of band emission and the continuous background; (iii) the absorption and emission components in composite bands; (iv) changes of equivalent widths of spectral lines with time; and (v) marked departures from black-body curves in the continuous spectrum. in $(b)$.

(c) Relations between variations in the light curve and the various items discussed

(d) Possible evidence for magnetic fields in the star or the shells.

(e) Pre-maximum spectra.

(2) (a) Spectral nomenclature. The separate details to be allowed for in the case of each component spectrum should include the following: (i) the stage of Nova development at which it is strongest; (ii) spectral type; (iii) nature of absorption lines (sharp, diffuse or very diffuse); (iv) at later stages the source of the spectrum: star or shell. 
(b) The physical relations between successive shells.

(c) The cause of the slowing down of the first pre-maximum spectrum.

(d) The possibility of measuring the brightness of the central star at different stages.

(e) The chemical abundance of elements.

(f) The evaluation of the total mass ejected by successive shells.

(g) The different distribution of different elements.

(h) The occurrence of condensations in pairs, generally equally displaced from the central star, in opposite directions.

(i) The asymmetry of the expanding envelope.

(j) The question of securing an atlas of the spectra of a series of Novae at different stages.

Supernovae. Supernovae are now subdivided into two classes (Minkowski, P.A.S.P. 55, I $94 \mathrm{I}$ ). Class I have the continuous spectrum very weak in the ultra-violet. There are a number of very wide emission bands, as yet unidentified (save for the possible presence of [O I] 6300,6363 as comparatively narrow bands at a late stage. To the violet of $\lambda_{5000}$ the bands shift gradually towards the red, while to the red of $\lambda 5000$ they appear and disappear much as bands do for ordinary novae: the spectra are closely comparable at the same time after maximum (Minkowski, $A p . J .59$, I56, I938). Class II show a strong extension into the ultra-violet for a week after maximum. Subsequently, absorptions and broad emission bands develop. Hydrogen and the usual forbidden lines are weak or missing, but the spectrum resembles that of a nova in the transition stage and may be built up by superposing a series of wide overlapping emission bands (Payne-Gaposchkin and Whipple). These are probably very bright and highly excited Novae of the ordinary type: there is reason to identify the Crab Nebula with a Nova of this type occurring some goo years ago. Nova Cassiopeiae $\mathrm{I}_{572}$ and Nova Ophiuchi I604 may have been class I supernovae in our galaxy.

Points to be discussed are the following: $(a)$ the spectra of class I stars; $(b)$ the stellar changes likely to accompany an outburst of the order of that of a supernova; $(c)$ the possible relation to white dwarfs.

\author{
F. J. M. Stratton \\ Chairman of the Sub-committee
}

\title{
VI. REPORT OF THE SUB-COMMITTEE ON MOLECULAR BANDS IN STELLAR SPECTRA
}

In preparing this report, the question arose as to the form which would be most useful. It was decided not to attempt to provide a bibliography of recent and current articles on molecular spectra, it being believed that the recent troubled conditions of the world have now subsided sufficiently to allow investigators to gain access to most, if not all, the important publications. Also, a complete bibliography would have made the report too long. It was decided that a useful means of encouraging and promoting the study of molecules in stellar atmospheres would be to list a number of problems in this field which are considered to be of importance at the present time. Accordingly, such a list is given below. To keep the report within reasonable length, only a limited number of problems can be described and those briefly. It is felt that a contribution to the solution of any one of them will be of definite value. The suggested problems have come from correspondence between the members of the sub-committee, a comprehensive and valuable memorandum by P. Swings being the first step in this process. Also, worthwhile suggestions have been received from H. D. Babcock, P. W. Merrill and R. F. Sanford of the Mount Wilson Observatory. 


\section{Observational Problems}

$x$. One of the problems requiring attention is the establishment of a physically significant sequence of the stars of type $S$. Their spectra are characterized by the occurrence of $\mathrm{ZrO}$ bands and the present sub-classification is ordered only upon the intensity of these bands. Whether this gives a true temperature scale has not been demonstrated and should be examined. The problem, complicated by some ambiguity in the distinction of type S from type $M$ stars, must, on account of the faintness and redness of the stars, be done using relatively low dispersion. Probably the red and perhaps the infra-red region should be included in the study. It is emphasized that the whole relationship of class S to class $M$ must be better understood before a completely satisfactory classification can be set up. Among the items that should be carefully studied are:

(a) Intensity of $\mathrm{ZrO}$ bands.

(b) Intensity of $\mathrm{TiO}$ bands.

(c) The excitation temperature indicated by the atomic line spectrum.

(d) The apparent abundance of various elements, especially $\mathrm{Y}, \mathrm{Zr}$ and $\mathrm{Ba}$.

2. It is suggested that observers be encouraged to make spectrographic observations as far as practicable into the infra-red for late-type stars. Both, photographic techniques, and those employing the new sensitive lead sulphide cells, could be used. In this region vibration-rotation bands of various molecules might be sought in the spectra of the coolest stars. As a low-dispersion application, extensive objective-prism surveys using Schmidttype telescopes and infra-red sensitive plates would supply useful information on the distribution of the faint cool stars.

3. A late M-type star and perhaps also a representative star of each of types $S, R$ and $\mathrm{N}$ should be studied with the highest practicable dispersion and in the greatest possible detail, as was recently done for $\beta$ Pegasi by Dorothy N. Davis $(A p . J$. ro6, 28, r947). Also, a detailed intercomparison, if necessary with more moderate but not low dispersion, should be made of the spectra of several stars of each of these types.

4. Observers of late-type stars should keep a close watch for the possible occurrence of bands due to polyatomic molecules, for example $\mathrm{CH}_{2}\left(\lambda_{4} \mathrm{O} 5 \mathrm{I}\right)$.

5. Observers of Novae should be alert to discover possible bands such as those of $\mathrm{C}_{2}$ and $\mathrm{CH}^{+}$as well as those of $\mathrm{CN}$ which have been noted previously. Occurrences of molecular bands in the spectra of novae are of much interest in connection with the behaviour of molecules in stellar atmospheres (e.g. questions of the photo-dissociation of molecules, see e.g. Swings and Struve, $A p . J .96,268-9$, I942).

6. A detailed study of the ultra-violet spectra of late-type stars under high dispersion would doubtless yield new and valuable information on molecular absorption features. For example, G. Herzberg has noted that on his recent plates of $\alpha$ Boo, the o, o and I, I NH bands show up much more clearly than in the previously published photographs which were secured in the pioneering work in this region. A specific problem is posed by the presence of unidentified bands in Se and Me spectra with heads near $\lambda \lambda 357 \mathrm{I}$ and 3680 reported by $\mathrm{P}$. W. Merrill.

7. The relative abundances in stellar atmospheres, of the isotopes of the elements is a question of much interest and of possible importance in connection with problems of energy generation, origin of the elements and stellar evolution. Measurements on molecular bands appear to be the only feasible method of attacking this subject. Data on the $\mathrm{C}^{12}$ to $\mathrm{C}^{13}$ ratio have been provided by a number of recent investigations. The most promising cases for study would appear to be the $\mathrm{TiO}$ and $\mathrm{ZrO}$ bands in $\mathrm{M}$ and S-type spectra. A careful study of these bands on high-dispersion spectrograms should enable valuable conclusions to be reached regarding the occurrence and possibly relative abundances of the isotopes of $\mathrm{Ti}, \mathrm{Zr}$ and $\mathrm{O}$.

8. It is worth while to emphasize that, in the study of molecules in stellar atmospheres, the normal solar and sunspot spectra offer valuable material for comparison. In connection with paragraph 2 above, H. D. Babcock and C. E. Moore in Carnegie Institution of Washington Publication, No. 579 (in the Press), on the Infra-red Solar Spectrum, give a

$$
296
$$


list of about 30 identified band heads between $\lambda 6600$ and $\lambda$ I3495. With reference to paragraph 6, H. D. Babcock, C. E. Moore and M. F. Coffeen in a forthcoming Mount Wilson contribution have revised the earlier data in the region $\lambda 2935$ to $\lambda 3063$ in the solar spectrum. Here, as well as having newly identified some $\mathrm{OH}$ and $\mathrm{NH}$ absorptions, they found several bands of unknown origin.

\section{EXPERIMENTAL WORK IN THE LABORATORY}

I. The experimental determination of the relative transition probabilities of the various bands within the systems occurring in stellar spectra would be of great value. The bandsystems include the violet and red $\mathrm{CN}$ bands, the Swan system of $\mathrm{C}_{2}$, the three systems of $\mathrm{CH}$, the three $\mathrm{TiO}$ systems and the $\mathrm{ZrO}$ systems. Possibly the best method would be the use of an electric furnace to produce the bands in absorption, employing the general techniques used by R. B. King and A. S. King to obtain $f$-values of atomic transitions (Ap. J. 87, 24, I938). Some work at present being done by R. B. King at the Mount Wilson Observatory will probably yield $f$-values for the $\mathrm{C}_{2}$ Swan bands.

2. A problem related to the above, namely determination of the absolute $f$-value of a molecular electronic transition (band-system as a whole) is also of interest, as applied to individual systems occurring in stellar spectra. Results could be applied to intensity measurements in stellar spectra to evaluate the relative numbers of absorbing molecules of different kinds. An $f$-value has been deduced for the violet $\mathrm{CN}$ bands by $\mathrm{J}$. $\mathrm{U}$. White (J.Chem. Phys. 8, 79 and 459, I940), whose method, or variations of it, might be applied to other molecules of astrophysical interest. The great need for studies of this type is illustrated by the discrepancies pointed out by $\mathrm{H}$. D. Babcock ( $A p . J$. 102, I65, I945) between different estimates of molecular abundances in the solar atmosphere.

3. Strong unidentified bands in the spectra of some N-type stars have been studied by R. F. Sanford, by C. D. Shane and recently by A. McKellar (J. Roy. Astr. Soc. Canada, 4I, I47, I947). Among possible carrier molecules suggested by the latter were $\mathrm{TiH}$ and FeH. G. Herzberg and J. G. Phillips have, during I947, tried a variety of sources in an attempt to produce in the laboratory the spectrum of $\mathrm{TiH}$, but without as yet any success. Further laboratory work on possible molecules is desirable. It is believed the blue-green bands have the distinction of being the strongest absorption features in stellar spectra as yet unidentified.

4. Detailed study of the band systems of $\mathrm{TiO}$ and $\mathrm{ZrO}$ as produced in the laboratory should yield data of value to astrophysicists. One particular aspect requiring attention is the isotope effect. Also, wave-lengths for the lines of many more bands of TiO than the three heretofore analysed should be measured and possibly certain details of the vibrational assignments checked. (See P. W. Merrill, Spectra of Long Period Variable Stars, University of Chicago Press, I940, ch. V.)

Three general remarks which apply not only to $\mathrm{TiO}$ and $\mathrm{ZrO}$ may be interposed at this point:

(a) Existing laboratory data on bands in the red and infra-red are very scanty, and it is in these regions that much new observational material on stellar spectra is being gathered.

(b) Most laboratory data relate to emission whereas the astrophysicist is more interested in absorption spectra.

(c) Physicists usually only measure the lines of enough bands to make possible the evaluation of the molecular constants. Measurements of all the intense bands of a system, admittedly a laborious process, would nevertheless be appreciated by astrophysicists.

It is hoped these remarks may be given consideration by spectroscopists doing work on bands in the laboratory.

5. Any laboratory investigation leading to more trustworthy and accurate values of the heats of dissociation of diatomic molecules involving astrophysically abundant elements is of great importance in making possible a more satisfactory calculation of the dissociation equilibrium in stellar atmospheres. 


\section{CALCULATION FROM THEORY}

I. It is very desirable that theoretical calculations of the relative intensities of the bands of the Swan system of $\mathrm{C}_{2}$, and also of the bands of the systems of $\mathrm{CN}, \mathrm{TiO}$ and $\mathrm{ZrO}$ be carried out. These should be as accurate as possible, using theory at least as refined as that given by E. Hutchisson for the anharmonic oscillator (Phys. Rev. 37, 45, I93I) in conjunction with the best values of the molecular constants. Such calculations would be a valuable check on and adjunct to the experimental determinations. When results such as these are available, reliable determinations of vibrational temperatures in stellar atmospheres can be found, independently of any possible curve-of-growth effect, from bands of similar intensity. Also information on the extent to which thermodynamic equilibrium obtains might be deduced. New relative transition probabilities based on the theory of the harmonic oscillator have been calculated for the violet $\mathrm{CN}$ and the Swan $\mathrm{C}_{2}$ systems by W. Buscombe and A. McKellar (Publ. Dom. Ap. Obs. 7, No. 23, in the Press), in connection with measurements of band intensities in R-type spectra.

2. Theoretical calculations of the absolute $f$-values of whole band systems of astrophysical interest are also of value, as indicated in paragraph 2 under experimental work. The agreement between theoretical and experimental values would allow an estimation of the accuracy of the $f$-values and possibly, too, of the theory.

3. Questions of the sources of opacity in stellar atmospheres, particularly in late-type stars, have recently become important. The significance of the role played by $\mathrm{H}^{-}$ions in solar-type stars has directed attention to negative ions. Certain molecules such as $\mathrm{O}_{2}$ can apparently form negative ions. Any calculations yielding results on the magnitude of the electron affinities of astrophysically abundant molecules or estimates of the effective absorptions of the ions would be received with the greatest interest.

\section{Compilation of Molecular Data}

r. A project, the results of which would prove of great value to astrophysicists interested in molecular bands, would be a compilation of an extensive table of molecular constants, etc. for all band systems of astronomical importance. Such a project could be a relatively long-term one. As well as the wave-lengths of the bands and their intensities, the various vibrational and rotational constants, the heats of dissociation, etc. should be given. Such a project might prove an interesting undertaking for some qualified person who need not necessarily have access to observational or laboratory equipment.

2. As a companion to the above, a photographic atlas of band spectra of astrophysical interest, reproduced in such a way that reasonably accurate wave-lengths could be read off easily, would be of much help to those studying the spectra of late-type stars. Such an atlas could best be prepared where both the laboratory facilities are available and, also, observational work on late-type stars is under way. The most suitable choice for this would appear to be the Mount Wilson Observatory, where laboratory spectra of most such molecular bands have already undoubtedly been photographed.

Both the above-mentioned projects might appropriately be done under the auspices of Commission 29. It is recommended therefore that the early preparation of the Atlas and Tables be encouraged and that, if necessary, some financial support be given by the International Astronomical Union.

ANDREW MCKELLAR Chairman of the Sub-commitlee

\section{RECOMMENDATION CONCERNING A SUB-COMMITTEE ON VARIABLE STARS}

Several members of this Commission (Merrill, Swings) have independently suggested that a sub-committee (originally proposed at the Stockholm meeting) be formed, consisting of members of this Commission and of Commission 27 (Variable Stars), in order 
to bring about prompt and effective collaboration between photometric and spectrographic observers. Commission 29 welcomes the spectacular development of accurate photoelectric methods and directs attention to several recent projects of collaboration (RR Lyrae: Leiden, photoelectric; McDonald, spectrographic). It wishes also to emphasize the great importance of maintaining accurate ephemerides of many eclipsing variables and of continuing the light curves of long-period and of irregular variables. These latter tasks can still be handled best by many observers with visual or simple photographic instruments.

\section{OtTo STRUve \\ President of the Commission}

\section{Report of meetings}

President: Prof. Otto Struve.

Secretary: Prof. Livio Gratton.

First meeting (August I2, I948. 9.30 a.m.)

I. List of stars for standard spectral types. The desirability that a list of standard stars for spectral types be made available as soon as possible, together with reproductions of standard spectra, was pointed out by Struve, Mrs Mayall, Swings and others. A committee was formed for discussing a preliminary list prepared by Joy and for adopting a definitive list. On the President's suggestion the following persons will be members of this informal committee: Vyssotsky (Chairman), Joy, Mrs Mayall, Morgan, Nassau, Schalén.

\section{Standard Stars for Spectral Type}

This list was originally composed by A. H. Joy of the Mount Wilson Observatory and presented to Commission 29, which passed a resolution that the list should be published after it had been discussed in detail by a small group appointed by the President of the Commission. The outcome of this discussion presents a compromise as to the choice of stars included. In general there is good agreement between the estimates made at Mount Wilson and Yerkes, with the exception, perhaps, of the dwarf $\mathrm{M}$ branch, where the Mount Wilson system was adopted for the present list.

The list is primarily intended for the use of observers with large instruments. To make it usable for smaller instruments, visual doubles were avoided as far as possible, or marked as VD when they were included. Similarly, a few spectroscopic binaries were marked as SB. It is assumed that an observer will wish to set up his own series of standard photographs taken with his particular instrument.

This list is subject to further revision if the need arises.

The following abbreviations have been used: $\mathrm{VD}=$ visual double; $\mathrm{SB}=$ spectroscopic binary; $\mathrm{P}=$ period; $d=$ distance; $\Delta m=$ difference in magnitudes; $\mathrm{Y}=$ Morgan, Keenan and Kellman, Atlas of Stellar Spectra.

\section{Wolf-Rayet Stars}

\begin{tabular}{|c|c|c|c|c|c|c|}
\hline $\begin{array}{l}\text { Spectral } \\
\text { type }\end{array}$ & Star & Boss & $\begin{array}{l}\text { Vis. } \\
\text { mag. }\end{array}$ & h. $\stackrel{\alpha}{\mathrm{m}}$. & $00 \delta$ & Remarks \\
\hline WN 5 & HD 187282 & $\ldots$ & $10 \cdot 0$ & $1946 \cdot 2$ & +184 & $\ldots$ \\
\hline WN 5 & HD 211564 & $\ldots$ & $11 \cdot 1$ & $2214 \cdot 7$ & +5521 & $\ldots$ \\
\hline WN 6 & HD 191765 & $\ldots$ & 7.8 & 208.4 & +361 & $\ldots$ \\
\hline WN 6 & HD 192163 & $\ldots$ & $7 \cdot 4$ & $2010 \cdot 3$ & +3812 & $\ldots$ \\
\hline WN 7 & HD 151932 & $\ldots$ & $6 \cdot 6$ & 1648.8 & -4546 & $\ldots$ \\
\hline WN 7 & HD 92740 & $\ldots$ & 6.5 & $1039 \cdot 3$ & -5925 & $\ldots$ \\
\hline WN 8 & HD 177230 & $\ldots$ & $11 \cdot 1$ & $\begin{array}{ll}19 & 1.3\end{array}$ & -424 & $\ldots$ \\
\hline \multirow[t]{2}{*}{ WN 8} & HD 96548 & $\cdots$ & $7 \cdot 8$ & $114 \cdot 3$ & -6514 & $\ldots$ \\
\hline & & & & 299 & & \\
\hline
\end{tabular}


Wolf-RAyet StaRs (continued)

Spectral type

WC6

WC6

WC7

WC7

WC8

WC8
Vis.

$\begin{array}{cr}\text { Boss } & \text { mag. } \\ \ldots & 10 \cdot 0 \\ \ldots & 7 \cdot 8 \\ \ldots & 7 \cdot 9 \\ \ldots & \mathbf{9 \cdot 4} \\ \ldots & 10 \cdot 0 \\ \ldots & 8 \cdot 8\end{array}$

h. $\mathrm{m}$

235.2

$18 \quad 5 \cdot 5$

2010.0

1339.5

1932.8

$1758 \cdot 4$ $\alpha$
$1950 \delta$,

$+5628$

$-2116$

$+363$

$-679$

$+3024$

$-3243$
Remarks

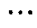

$\cdots$

$\cdots$

...

$\cdots$

\section{Giants}

\begin{tabular}{|c|c|c|c|}
\hline O5 & $9 \mathrm{Sgr}$ & 4560 & 5.9 \\
\hline O6 & HD 190864 & $\ldots$ & $8 \cdot 2$ \\
\hline O7 & 9 Sge & 5073 & $6 \cdot 3$ \\
\hline 08 & $\lambda^{1}$ Ori & $1357 \mathrm{~A}$ & $3 \cdot 7$ \\
\hline Bo & $v$ Ori & 1340 & $4 \cdot 6$ \\
\hline Bo & $\kappa$ Ori & 1435 & $2 \cdot 2$ \\
\hline BI & $\beta \mathrm{CMa}$ & 1609 & $2 \cdot 0$ \\
\hline B2 & $12 \mathrm{Lac}$ & 5856 & $5 \cdot 2$ \\
\hline B3 & GC 6632 & 1295 & $5 \cdot 6$ \\
\hline В5 & $18 \mathrm{Peg}$ & 5653 & $6 \cdot 0$ \\
\hline B5 & $\delta$ Per & 838 & $3 \cdot 1$ \\
\hline B8 & $\beta$ Tau & 1304 & 1.8 \\
\hline B9 & $\gamma \mathrm{Lyr}$ & 4824 & $3 \cdot 3$ \\
\hline A0 & GC 27939 & 5160 & $6 \cdot 2$ \\
\hline A. 0 & $\delta$ Cyg & 5048 & $3 \cdot 0$ \\
\hline A2 & $\beta$ Ser & 4009 & $3 \cdot 7$ \\
\hline A2 & $\lambda \mathrm{UMa}$ & 2729 & $3 \cdot 5$ \\
\hline A3 & $\delta$ Her & 4376 & $3 \cdot 2$ \\
\hline A5 & $\beta$ Tri & 482 & $3 \cdot 1$ \\
\hline A5 & $\alpha \mathrm{Oph}$ & 4459 & $2 \cdot 1$ \\
\hline A 7 & $\gamma \mathrm{Boo}$ & 3722 & $3 \cdot 0$ \\
\hline Fo & $\zeta$ Leo & 2730 & $3 \cdot 7$ \\
\hline $\mathrm{F} 2$ & $\beta$ Cas & 12 & $2 \cdot 4$ \\
\hline F2 & $20 \mathrm{Peg}$ & 5658 & $5 \cdot 7$ \\
\hline F5 & `Vir & 3660 & $4 \cdot 2$ \\
\hline F5 & 25 Mon & 1999 & $5 \cdot 2$ \\
\hline F 8 & $1 \mathrm{Com}$ & 3142 & $6 \cdot 6$ \\
\hline $\mathrm{G} 2$ & $\eta \mathrm{Peg}$ & 5865 & $3 \cdot 1$ \\
\hline G5 & $\gamma$ Hya & 3449 & $3 \cdot 3$ \\
\hline G8 & $\epsilon \mathrm{Oph}$ & 4147 & $3 \cdot 3$ \\
\hline G8 8 & $\kappa$ Cyg & 4923 & $4 \cdot 0$ \\
\hline Ko & $\eta$ Cyg & 5103 & $4 \cdot 0$ \\
\hline KO & $\nu \mathrm{Oph}$ & 4536 & $3 \cdot 5$ \\
\hline K2 & $\kappa \mathrm{Oph}$ & 4315 & $3 \cdot 4$ \\
\hline K5 & GC 33081 & 6118 & $5 \cdot 9$ \\
\hline K5 & $\gamma$ Dra & 4541 & $2 \cdot 4$ \\
\hline Mo & $\beta$ And & 259 & $2 \cdot 4$ \\
\hline MI & 7 Cet & 33 & $4 \cdot 7$ \\
\hline MI & 75 Cyg & 5567 & $5 \cdot 4$ \\
\hline M2 & $\alpha$ Cet & 691 & $2 \cdot 8$ \\
\hline M2 & $x$ Peg & 31 & $4 \cdot 9$ \\
\hline M3 & $\hat{\mu} \mathrm{Gem}$ & 1604 & $3 \cdot 2$ \\
\hline M4 & GC 265 & 30 & $5 \cdot 4$ \\
\hline M5 & 56 Leo & 2915 & $6 \cdot 0$ \\
\hline M6 & 45 Ari & 660 & $5 \cdot 9$ \\
\hline 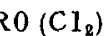 & HD 182040 & $\ldots$ & $7 \cdot 0$ \\
\hline & HD 16115 & & 3 \\
\hline
\end{tabular}

$\begin{array}{rr}180 \cdot 8 & -2422 \\ 203 \cdot 8 & +3528 \\ 1950 \cdot 1 & +1833 \\ 532 \cdot 4 & +954 \\ 529 \cdot 5 & -720 \\ 545 \cdot 4 & -941 \\ 620 \cdot 5 & -1756 \\ 2239 \cdot 2 & +3958 \\ 521 \cdot 1 & -012 \\ 2157 \cdot 6 & +629 \\ 339 \cdot 4 & +4738 \\ 523 \cdot 1 & +2834 \\ 1857 \cdot 1 & +3237 \\ 205 \cdot 8 & -1013 \\ 1943 \cdot 4 & +450 \\ 1543 \cdot 9 & +1535 \\ 1014 \cdot 1 & +4310 \\ 1713 \cdot 0 & +2454 \\ 266 \cdot 6 & +3445 \\ 1732 \cdot 6 & +1236 \\ 1430 \cdot 1 & +3832 \\ 1013 \cdot 9 & +2340 \\ 06 \cdot 5 & +5852 \\ 2158 \cdot 7 & +1253 \\ 1413 \cdot 4 & -546 \\ 734 \cdot 8 & -40 \\ 1159 \cdot 2 & +2222 \\ 2240 \cdot 7 & +2958 \\ 1316 \cdot 2 & -2255 \\ 1615 \cdot 7 & -434 \\ 1915 \cdot 9 & +5317 \\ 1954 \cdot 4 & +3457 \\ 1756 \cdot 3 & -946 \\ 1655 \cdot 3 & +927 \\ 2348 \cdot 0 & -1441 \\ 1755 \cdot 4 & +5130 \\ 16 \cdot 9 & +3521 \\ 012 \cdot 1 & -1913 \\ 2138 \cdot 2 & +433 \\ 259 \cdot 7 & +354 \\ 012 \cdot 0 & +1956 \\ 619 \cdot 9 & +2232 \\ 011 \cdot 9 & -84 \\ 1053 \cdot 4 & +627 \\ 253 \cdot 0 & +188 \\ 1920 \cdot 4 & -1048 \\ 232 \cdot 7 & -940 \\ & \end{array}$

300 


\section{GIANTS (continued)}

\begin{tabular}{|c|c|c|c|c|c|c|}
\hline $\begin{array}{l}\text { Spectral } \\
\text { type }\end{array}$ & Star & Boss & $\begin{array}{l}\text { Vis. } \\
\text { mag. }\end{array}$ & h. $\stackrel{\alpha}{\mathrm{m}}$. & $1950 \delta$, & Remarks \\
\hline $\mathrm{R} 6\left(\mathrm{C} 4_{4}\right)$ & HD 52434 & .. & $7 \cdot 5$ & $658 \cdot 5$ & -311 & $\ldots$ \\
\hline $\mathrm{R} 8\left(\mathrm{C}_{3}\right)$ & HD 25408 & & 7.9 & $\begin{array}{ll}4 & 1.5\end{array}$ & +6140 & $\cdots$ \\
\hline $\mathrm{N} 0\left(\mathrm{C}_{2}\right)$ & 19 Psc & 6102 & $5 \cdot 3$ & $2343 \cdot 8$ & +313 & $\cdots$ \\
\hline $\mathrm{N2}\left(\mathrm{C}_{3}\right)$ & U Hya & 2827 & $5-6$ & $1035 \cdot 1$ & -137 & $\ldots$ \\
\hline $\mathrm{N} 6 \mathrm{e}\left(\mathrm{C} 7_{4}\right)$ & R Lep & $\ldots$ & $6-10$ & $457 \cdot 3$ & -1453 & $\ldots$ \\
\hline $\mathrm{Se}(\mathrm{S} 2 \mathrm{e})$ & V Cnc & $\ldots$ & $7-13$ & $818 \cdot 8$ & +1726 & $\ldots$ \\
\hline Se (S3e) & R Gem & $\ldots$ & $6-14$ & $\begin{array}{ll}7 & 4 \cdot 4\end{array}$ & +2247 & $\ldots$ \\
\hline Se (S3e) & R Cyg & $\cdots$ & $6-14$ & 1935.5 & +505 & $\ldots$ \\
\hline $\mathrm{Se}(\mathrm{S} 4 \mathrm{e})$ & R And & $\ldots$ & $6-15$ & $021 \cdot 4$ & +3818 & $\ldots$ \\
\hline $\mathrm{S}(\mathrm{S} 4)$ & HD 35155 & $\ldots$ & $7 \cdot 0$ & 519.9 & -843 & $\ldots$ \\
\hline
\end{tabular}

Note. Second spectra of carbon stars are as given by Keenan and Morgan $(A P . J .94,501)$, and those of ZrO stars are due to Dorothy N. Davis (P.A.S.P. 46, 267).

\begin{tabular}{|c|c|c|c|c|c|c|c|}
\hline \multicolumn{8}{|c|}{ Main Sequence } \\
\hline 06 & $\theta^{\prime}$ Ori C & 1363 & $5 \cdot 4$ & $532 \cdot 8$ & -525 & & $\ldots$ \\
\hline 06 & $\lambda C_{\mathrm{ep}}$ & 5719 & $5 \cdot 2$ & $\begin{array}{ll}22 & 9 \cdot 8\end{array}$ & +5910 & & \\
\hline O9 & ¿Ori & $1366 \mathrm{br}$. & $2 \cdot \overline{9}$ & $533 \cdot 0$ & -556 & $\mathrm{SB}: \mathrm{P}=29^{d}$ & \\
\hline BO & $\zeta \mathrm{Oph}$ & 4225 & 2.7 & $1634 \cdot 4$ & -1028 & & \\
\hline B1 & $\eta$ Ori & 1301 & $3 \cdot 4$ & $522 \cdot 0$ & -226 & $\begin{array}{r}\mathrm{SB}: \mathrm{P}=8^{d} \\
\Delta m=\mathrm{l}^{\mathrm{m} \cdot 0}\end{array}$ & ${ }_{0}^{\mathrm{VD}:} d=1^{\prime \prime}$, \\
\hline B2 & ${ }_{\nu} \mathrm{Sco}$ & 4117 & $4 \cdot 3$ & $\begin{array}{ll}16 & 9 \cdot 1\end{array}$ & -1920 & $\mathrm{VD}: d=1^{\prime \prime}$ & $\Delta m=2^{\mathrm{m} \cdot 5}$ \\
\hline $\mathrm{B} 2$ & GC 28228 & 5210 & $5 \cdot 8$ & $2016 \cdot 4$ & +4035 & $\mathrm{VD}: d=3^{\prime \prime}$ & $\Delta m=2^{\mathrm{m}} \cdot 2$ \\
\hline B3 & $\mu$ Aur & I204 & $3 \cdot 3$ & $5 \quad 3.0$ & +4110 & & $\ldots$ \\
\hline B3 & GC 23058 & 4345 & $5 \cdot 6$ & $17 \quad 3 \cdot 0$ & -050 & & $\ldots$ \\
\hline B5 & $\kappa \mathrm{Hya}$ & 2600 & $5 \cdot 0$ & 937.9 & -146 & & $\ldots$ \\
\hline B8 & $\beta \mathrm{Lib}$ & 3890 & $2 \cdot 7$ & $1514 \cdot 3$ & -912 & & $\ldots$ \\
\hline B9 & $\alpha \mathrm{Peg}$ & 5944 & $2 \cdot 6$ & $\begin{array}{ll}23 & 2 \cdot 3\end{array}$ & +1456 & & $\ldots$ \\
\hline A 0 & 30 Mon & 2237 & $4 \cdot 0$ & $823 \cdot 2$ & -345 & & $\ldots$ \\
\hline A 2 & $\zeta \mathrm{Vir}$ & 3508 & $3 \cdot 4$ & $1332 \cdot 1$ & -020 & $\mathrm{Y}: \mathrm{A} \mathbf{3}$ & \\
\hline A 5 & $\beta$ Ari & 428 & $2 \cdot 7$ & $151 \cdot 9$ & +2034 & $\mathrm{SB}: \mathrm{P}=107^{\circ}$ & \\
\hline A 7 & $\alpha \mathrm{Aql}$ & 5062 & 0.9 & 1948.3 & $\begin{array}{r}+844 \\
\end{array}$ & & $\ldots$ \\
\hline A 8 & $53 \mathrm{Her}$ & 4300 & $5 \cdot 4$ & $1651 \cdot 1$ & +3147 & $\mathrm{Y}: \mathrm{F} 2$ & \\
\hline Fo & $\mu$ Cap & 5623 & $5 \cdot 2$ & $2150 \cdot 6$ & -1347 & & $\ldots$ \\
\hline $\mathrm{F} 2$ & $\sigma$ Boo & 3729 & $4 \cdot 5$ & $1432 \cdot 5$ & +2958 & & $\ldots$ \\
\hline F5 & $\gamma$ Ser & 4055 & $3 \cdot 9$ & $1554 \cdot 1$ & +1549 & Y: F6 IV & \\
\hline F5 & ¿Peg & 5688 & $4 \cdot 0$ & $\begin{array}{ll}22 & 4.7\end{array}$ & +256 & & $\ldots$ \\
\hline $\mathrm{F} 8$ & $\beta \mathrm{Vir}$ & 3105 & $3 \cdot 8$ & $1148 \cdot 1$ & $\begin{array}{r}+23 \\
\end{array}$ & & $\ldots$ \\
\hline Go & $\delta$ Tri & 514 & $5 \cdot 4$ & 214.0 & +340 & $\mathrm{SB}: \mathrm{P}=10^{d}$ & \\
\hline G0 & $\lambda$ Ser & 4010 & $4 \cdot 4$ & 1544.0 & $\begin{array}{l}731 \\
+73\end{array}$ & $\mathrm{Y}: \mathrm{G} 2$ & \\
\hline $\mathrm{G} 2$ & $72 \mathrm{Her}$ & 4403 & $5 \cdot 4$ & 1718.8 & +3232 & & $\ldots$ \\
\hline G5 & $\kappa$ Cet & 752 & $5 \cdot 0$ & 316.7 & +311 & & $\ldots$ \\
\hline G8 8 & $\tau$ Cet & 391 & $3 \cdot 7$ & $141 \cdot 7$ & -1612 & & $\ldots$ \\
\hline G8 & GC 95 & 6 & $6 \cdot 2$ & $\begin{array}{lll}0 & 4.0\end{array}$ & +2845 & & $\ldots$ \\
\hline Ko & $54 \mathrm{Psc}$ & 133 & $6 \cdot 1$ & 036.8 & $\begin{array}{r}+2059 \\
\end{array}$ & & $\ldots$ \\
\hline $\mathrm{K} 2$ & $\epsilon$ Eri & 814 & $3 \cdot 8$ & $330 \cdot 6$ & -938 & & $\ldots$ \\
\hline $\mathrm{K} 4$ & GC 32089 & $\ldots$ & $7 \cdot 6$ & $2259 \cdot 3$ & -47 & & $\ldots$ \\
\hline K 6 & 61 Cyg A & 5433 & $5 \cdot 6$ & $21 \quad 4 \cdot 7$ & +3830 & $\mathrm{Y}: \mathrm{K} 5$ & \\
\hline M0 & 61 Cyg B & 5434 & $6 \cdot 3$ & $21 \quad 4 \cdot 7$ & +3830 & & $\ldots$ \\
\hline MO & $\mathrm{C} 1412$ & $\ldots$ & $9 \cdot 7$ & $1125-9$ & $\begin{array}{r}750 \\
\end{array}$ & Sf of two sta & ars \\
\hline Ml & $\mathrm{BD}+42^{\circ} 2296$ & $\ldots$ & $9 \cdot 1$ & $1219 \cdot 4$ & +4226 & & $\ldots$ \\
\hline M1 & $\mathrm{BD}-2 \mathrm{I}^{\circ} 1051$ & $\ldots$ & $8 \cdot 3$ & $\begin{array}{ll}5 & 0 \cdot 3\end{array}$ & -2120 & & $\ldots$ \\
\hline M2 & $\mathrm{BD}+36^{\circ} 2147$ & 2935 & $7 \cdot 6$ & 110.6 & +3618 & & $\ldots$ \\
\hline M3 & $\mathrm{BD}+4^{\circ} 4048$ & $\ldots$ & $9 \cdot 2$ & $1914 \cdot 5$ & +56 & & $\ldots$ \\
\hline & & & & $30 I$ & & & \\
\hline
\end{tabular}




\section{MaIn Sequence (continued)}

Spectral

type

M4

M4

M5

M5

M6e
Vis.

Star Boss mag.

GC 25648 A

$\mathrm{BD}+5^{\circ} 1168$

$\mathrm{BD}-12^{\circ} 4523$

$\mathrm{BD}+4^{\circ} 3561$

$20 \mathrm{C} 600$ $\begin{array}{rr}\ldots & 8 \cdot 9 \\ \ldots & 10 \cdot 0\end{array}$

... $\quad 10 \cdot 0$

... 9.5

... $13 \cdot 5$ h. ${ }^{\alpha} 1950 \delta_{0}^{\delta}$,

$1842 \cdot 2+5933$

$724 \cdot 7+530$

$1627.5 \quad-1232$

$1755.4+424$

$1054 \cdot 2+720$
Remarks

$\mathrm{VD}: d=17^{\prime \prime}, \Delta m=0^{\mathrm{m}} \cdot 8$

\section{SUPER-GIANTS}

\begin{tabular}{|c|c|c|c|c|c|c|}
\hline 09 & HD 188209 & 5083 & $\mathbf{5} \cdot \mathbf{5}$ & 1950.5 & +4654 & $\cdots$ \\
\hline O9 & $16 \mathrm{Sgr}$ & 4613 & $6 \cdot 0$ & $1812 \cdot 2$ & -2024 & Two spectra; rad. vel. var. \\
\hline Bo & $\epsilon$ Ori & 1370 & $1 \cdot 8$ & $533 \cdot 7$ & -114 & $\ldots$ \\
\hline B1 & $\zeta$ Per & 894 & $2 \cdot 9$ & $351 \cdot 0$ & +3144 & $\ldots$ \\
\hline $\mathrm{B2}$ & $\chi^{2}$ Ori & 1507 & $4 \cdot 7$ & 60.9 & +208 & $\ldots$ \\
\hline B3 & 9 Gem & 1578 & $6 \cdot 3$ & 613.9 & +2346 & $\ldots$ \\
\hline B3 & $55 \mathrm{Cyg}$ & 5361 & $4 \cdot 9$ & $2047 \cdot 2$ & +4556 & $\ldots$ \\
\hline B5 & $67 \mathrm{Oph}$ & 4548 & $3 \cdot 9$ & $1758 \cdot 1$ & +256 & $\ldots$ \\
\hline $\mathrm{B} 8$ & $\beta$ Ori & 1250 & $0 \cdot 3$ & $512 \cdot 1$ & -815 & $\mathrm{SB}: \mathrm{P}=\mathbf{2 2}^{d}$ \\
\hline AO & $\eta$ Leo & 2694 & $3 \cdot 6$ & $10 \quad 4 \cdot 6$ & +170 & .. \\
\hline A 2 & $\alpha$ Cyg & 5320 & $1 \cdot 3$ & $2039 \cdot 7$ & +456 & $\ldots$ \\
\hline A5 & $\phi$ Cas & 298 & $5 \cdot 3$ & 116.9 & +5758 & ... \\
\hline A 7 & 97 Pup & 1968 & $4 \cdot 8$ & $727 \cdot 7$ & -2255 & $\ldots$ \\
\hline A 8 & GC 24543 & $\cdots$ & $7 \cdot 3$ & $1759 \cdot 5$ & -2254 & ... \\
\hline F0 & $\alpha$ Lep & 1347 & $2 \cdot 7$ & 530.5 & -1751 & $\cdots$ \\
\hline $\mathrm{F} 2$ & $\pi \mathrm{Sgr}$ & 4874 & $3 \cdot 0$ & $19 \quad 6 \cdot 8$ & -216 & ... \\
\hline F3 & 10 Pup & 2082 & $5 \cdot 7$ & $750 \cdot 0$ & -1443 & $\ldots$ \\
\hline F4 & $\nu \mathrm{Aql}$ & 4962 & $4 \cdot 9$ & $1924 \cdot 0$ & +014 & $\cdots$ \\
\hline F5 & $\alpha$ Per & 772 & $1 \cdot 9$ & $320 \cdot 7$ & +4941 & $\cdots$ \\
\hline F 8 & $\gamma$ Cyg & 5229 & $2 \cdot 3$ & $2020 \cdot 4$ & +406 & $\ldots$ \\
\hline G0 & $\beta \mathrm{Aqr}$ & 5527 & $3 \cdot 1$ & 2128.9 & -548 & $\cdots$ \\
\hline G0 & GC 30534 & 5612 & $7 \cdot 2$ & $2146 \cdot 2$ & +3843 & $\ldots$ \\
\hline G1 & $\alpha$ Aqr & 5676 & $3 \cdot 2$ & $22 \quad 3 \cdot 2$ & -034 & $\cdots$ \\
\hline G2 & 11 Pup & 2099 & $4 \cdot 4$ & $754 \cdot 7$ & -2245 & $\cdots$ \\
\hline G3 & $\omega$ Gem & 1806 & $5 \cdot 2$ & $659 \cdot 4$ & +2417 & $\cdots$ \\
\hline G3 & GC 32063 & 5931 & $5 \cdot 5$ & $2258 \cdot 0$ & +5641 & ... \\
\hline G4 & $\zeta$ Cap & 5507 & $3 \cdot 9$ & $2123 \cdot 8$ & -2238 & $\cdots$ \\
\hline G5 & $9 \mathrm{Peg}$ & 5590 & $4 \cdot 5$ & $2142 \cdot 1$ & +177 & ... \\
\hline G8 & E Gem & 1717 & $\mathbf{3 \cdot 2}$ & $640 \cdot 9$ & +2511 & $\cdots$ \\
\hline KO & $56 \mathrm{Peg}$ & 5954 & $5 \cdot 0$ & $23 \quad 4 \cdot 7$ & +2512 & $\mathrm{Y}: \mathrm{G} 8, \mathrm{H}+\mathrm{K}$ in emission \\
\hline $\mathrm{K} 1$ & $\zeta$ Cep & 5714 & $3 \cdot 6$ & $22 \quad 9 \cdot 1$ & +5757 & ... \\
\hline $\mathbf{K 2}$ & 56 Ori & 1458 & $5 \cdot 0$ & $549 \cdot 8$ & +151 & ... \\
\hline K3 & 10 Ori & 1181 & $4 \cdot 7$ & $456 \cdot 0$ & +138 & ... \\
\hline K4 & $\gamma \mathrm{Aql}$ & 5047 & $2 \cdot 8$ & $1943 \cdot 9$ & +1029 & $\ldots$ \\
\hline K5 & 3 Cet & 6182 & $5 \cdot 2$ & $\begin{array}{lll}0 & 1.9\end{array}$ & -1047 & $\cdots$ \\
\hline M1 & $\alpha \operatorname{Sco}$ & 4193 & $1 \cdot 2$ & $1626 \cdot 3$ & -2619 & $\cdots$ \\
\hline M2 & $\alpha$ Ori & 1468 & 0.9 & $552 \cdot 5$ & +724 & ... \\
\hline M3 & $\pi$ Aur & 1479 & $4 \cdot 6$ & $556 \cdot 2$ & +4556 & ... \\
\hline M5 & $\alpha$ Her & 4373 & $3 \cdot 6$ & $1712 \cdot 4$ & +1427 & $\mathrm{VD}: d=5^{\prime \prime}, \Delta m=2 \mathrm{~m} \cdot 0$ \\
\hline
\end{tabular}

Note. According to the Yerkes observers, the last two stars are not of the highest luminosity among super-giants; the following variables are examples of $M$ type stars of highest luminosity:

$\begin{array}{rrrrrrr}\text { M3-M4 } & \text { SU Per } & \ldots & 7-9 & 218 \cdot 6 & +5623 \\ \text { M5-M6 } & \text { RS Cnc } & \ldots & 5-7 & 97 \cdot 6 & +31 \\ \text { M5-M6 } & \text { Y Lyn } & \ldots & 7-8 & 724 \cdot 6 & +46 & 5\end{array}$


2. Classification of white dwarfs and sub-dwarfs. The president read a letter by Dr Luyten on the spectral classification of stars below the main sequence. After a discussion by Swings, Gratton, Vyssotsky and others, the Commission recommended that a summary of Dr Luyten's letter be prepared by Gratton and Vyssotsky and included in the Report.

After calling attention to the necessity of a more logical system of classification of stars below the main sequence, Dr Luyten pointed out that the designation of very dense stars as dwarfs or intermediate stars as sub-dwarfs is inadequate, because in some cases these stars might be more luminous than main sequence stars of later spectral types. Therefore, he suggested that the prefix 'sd' should be dropped. Instead of that he proposed to denote the bona fide white dwarfs and degenerate stars by using the letter $\mathrm{D}$ followed by a symbol of spectral class, such as DA; if the spectrum is virtually continuous, the spectral type should be designated as DC. For intermediate (sub-dwarfs) stars he proposes to postpone the final decision.

3. Sub-committee on the spectra of variable stars. After a suggestion by Gratton, Swings and Stratton, the following resolution was adopted:

Commission 29 recommends to the Executive Committee that a Sub-Commission on the spectra of all variable stars be created; that Commission 27 (Var. Stars) furnish two or three of its members and Commission 29 (Stellar Spectra) an equal number.

4. Report of the President of the Sub-committee of Novae. In addition to the Draft Report, Stratton read an important paper by McLaughlin containing an outline of a classification of the spectra of Novae. As the paper is too long for publication in the Report, it is recommended that McLaughlin's ideas be considered in preparing a scheme of a classification of the spectra of Novae spectra; this should be especially considered in the preparation of an Atlas of representative spectra of Novae. The following persons were designated to co-operate in the preparation of a new Atlas: Stratton (acting as Chairman), Bertaud, McLaughlin. Stratton, Bertaud and Gratton will prepare during the meeting a scheme of classification which could serve as a basis for the Atlas and would include the essentials of the suggestions contained in McLaughlin's letter (see § I3) Stratton then read a letter by Dr Baade of which the following is a summary as prepared by Gratton and Stratton.

Dr Baade's letter contained the important suggestion that the well-known features of the complex nebular lines in Nova Aq I I9I8 arise from rings instead of from shells or jets. Baade calculates from the observations a complete system of rings parallel to the equator and symmetrically placed north and south; the inclination of the polar axis of the rotating star being at $15^{\circ}$ to the line of sight. Although it is not easy to understand the physical relationship between these rings and the rotational state of the star, Baade's idea is very interesting and may have application to the interpretation of the spectral features of Novae.

Finally, the Sub-Commission recommended that those observers who are not continuously working on Novae and possess valuable observational material should lend it to other workers in this field. It was mentioned that copies of the Atlas of the Spectrum of Nova Her 1934 are still available.

5. Standard areas for spectral classification. A. N. Vyssotsky recommended the adoption of ten standard areas in which a number of stars should be classified as to luminosity and temperature. Miss M. E. Walther and Nassau remarked that some of these areas have been taken care of, while the others might not be suitable. Vyssotsky replied that the list is flexible and may be modified. The Commission recommended that the list is to be adopted as printed in the Draft Report.

6. Researches on stellar spectra in Japan. The following is a summary of a letter by Dr Hagihara, read by the President of the Commission.

Dr Hagihara quoted some interesting results by Japanese astronomers concerning mainly the chemical composition and structure of stellar atmospheres. These relate to the branching of the spectral sequence at types M-S (Fujita); to the abundance of $\mathrm{C}$ and $\mathrm{N}$ in some later type stars; to the computation of model atmospheres (Miyamoto); to 
Unsöld's theory of convective equilibrium taking into account the ionization (Hitotuyanagi); and to the theory of the atmospheres of Be stars. Finally, colour observations of Nova Pup 1942 by Tonita and Haruhata are also mentioned.

At Ir.30 a.m. the meeting was adjourned.

\section{Second meeting (August $\mathrm{x} 4$, I948. 9.15 a.m.)}

7. Molecular spectra. Dr Swings underlined the importance of work in the field of molecular spectra. The need of a compilation of molecular data was especially felt; an atlas containing the spectra of molecules of astrophysical interest would be extremely useful. Dr Swings suggested that the Atlas might possibly be prepared at the Vatican Observatory in collaboration with other institutions.

Dr Rosen stated that a compilation of all existing molecular data is being prepared by the International Chemical Union (Tables of Constants) and that it would be important to organize a collaboration between the Astronomical and Chemical Unions in order to save effort and avoid duplication of work. The needs of both organizations are closely related to each other. Moreover, it is difficult to decide on the kinds of molecules which are or may become in the future of astrophysical interest. Following a suggestion of the International Chemical Union, it may be possible to publish the table as a part of the 'Tables of Constants'. Father Junkes pointed out some difficulties concerning the preparation of the Atlas at the Vatican Observatory but gave assurance of interest on behalf of that institution. Accordingly the following informal committee was appointed for the compilation of molecular data and the preparation of the Atlas: McKellar, Junkes (or Gatterer), Herzberg, R. B. King, R. W. B. Pearse, Rosen, Swings.

The following recommendation was then adopted: 'Commission 29 recommends that a compilation and an atlas of laboratory molecular spectra of astrophysical interest be prepared and published as soon as possible.'

8. General report. Commission 29 voted that the Draft Report be adopted as printed, without further discussion.

9. Photography of the sky with large-field telescopes and objective prisms. Vyssotsky pointed out the importance of Schwassmann's plan for the complete photography of the whole sky with large field telescopes and objective prisms. After a short discussion by Struve, Gratton and Nassau, the Commission adopted the following resolution:

Commission 29 recommends that Prof. Schwassmann's plan to photograph the whole sky with large Schmidt telescopes equipped with an objective prism be followed as far as possible. If several observatories co-operate in this plan, periodic reports and exchange of information will be necessary. The President of Commission 29 will serve as an intermediary for that purpose.

The following table was prepared from information available at the meeting:

\begin{tabular}{|c|c|c|c|c|}
\hline Observatory & $\begin{array}{c}\text { Person in } \\
\text { charge }\end{array}$ & Region covered & Dispersion & $\begin{array}{l}\text { Limiting } \\
\text { magnitude }\end{array}$ \\
\hline McCormick & Vyssotsky & $+90^{\circ}$ to $-15^{\circ}$ & $300 \mathrm{~A} . / \mathrm{mm}$. & 12 \\
\hline Bergedorf & Wachmann & North sel. areas & ," & 13 \\
\hline Potsdam & $?$ & South sel. areas & $"$ & 12 \\
\hline Case & Nassau & Selected regions & $280 \mathrm{~A} . / \mathrm{mm}$. & $12 \cdot 5$ \\
\hline Tonanzintla & Erro & $?$ & " & $12 \cdot 5$ \\
\hline Harvard & Mayall & $+20^{\circ}$ to $-90^{\circ}$ & $400 \mathrm{~A} . / \mathrm{mm}$. & $11 \cdot 5$ \\
\hline Palomar & Zwicky & ? & $?$ & $12 \cdot 5$ \\
\hline Vatican & - & - & - & - \\
\hline Stockholm & - & - & - & - \\
\hline
\end{tabular}

Io. Mrs Mayall's report on spectral classification. The work of the Henry Draper Extension is being continued at Harvard and the next volume will be published in the early fall of this year. It will contain all of Miss Cannon's unpublished spectrum classifications, supplemented by a few of mine, about 85,000 stars in all. My current programme 
is a large Milky Way region extending south from Monoceros. I am continuing my Sagittarius nova patrol programme, and have a spectrum plate taken about once a month during the observing season on each of three selected centres in the Sagittarius-Scorpius cloud. I am also having spectrum plates taken to cover the whole southern sky, from $+20^{\circ}$ to $-90^{\circ}$, limiting magnitude about II.5.

Ir. Mrs Burbidge suggests investigations of all Be stars which show some changes for the purpose of studying magnetic fields.

I2. Mlle Bloch reports on recent observations of Nova Ser I 948 and Nova Cygni I948 at l'Observatoire St Michel. A sudden change in the spectrum of Nova Ser I948 on June 16 is especially interesting; unfortunately, Mlle Bloch's observations at that time have large gaps; hence it would be very important to know whether other observers possess spectra of the Nova at about that time.

13. Fehrenbach reports on a method of measuring radial velocities by means of an objective prism; his results are very promising and the method constitutes a great improvement over previous ones.

I4. A scheme of classification of the spectra of Novae. The lack of a uniform and generally understood nomenclature of the systems of lines and the stages of development of the spectrum of a Nova is a very great difficulty that is encountered by all stellar spectroscopists working on that field. This is probably due to the fact that each star has been studied in detail by a different investigator, and the manner of presentation of the results has been therefore different in each case. Now several good records of the spectra of different Novae are available and enough is known about the normal sequence of stages to justify an attempt at a general classification.

In attempting a classification of the spectra of Novae, it has to be borne in mind that often these spectra are very complex in the sense that at the same time several sets or systems of lines are present both in absorption and emission, which are distinguished from each other by a common radial velocity, excitation potential, etc. These systems differ somewhat in different Novae, but they have, nevertheless, some common features which permit one to recognize them and justify the use of the same designation. It is suggested that Roman numerals be used to indicate the systems of lines, leaving the general letter $Q$ followed by a number for the designation of the more or less complex spectrum which results from the superposition of several systems of lines and is characteristic of each stage of development. The following systems seem to be normal in ordinary Novae:

Premaximum
Principal
Diffuse Enhanced
Orion
Nitrogen III $(\lambda 4640)$
$\eta$ Carinae (forbidden Fe II)
Nebular
Wolf-Rayet

\begin{tabular}{ll} 
Absorption I & Emission I \\
Absorption II & Emission II \\
Absorption III & Emission III \\
Absorption IV & Emission IV \\
Absorption V & Emission V \\
- & Emission VI \\
- & Emission VII \\
\hline & Emission VIII
\end{tabular}

Systems I and II often closely resemble spectra of normal stars (usually super-giants); in these cases the spectral type may be added in parentheses, for instance II ( $\mathrm{F} 5$ ) is a fair and short description of the principal spectrum of N Her I934. Absorption III consists of a few rather broad lines of hydrogen and ionized metals, and absorption IV (usually indicated as ' Orion spectrum') consists of lines of N II, O II, C II, He I, etc. These absorption systems are often multiple, consisting of several components of different radial velocities. For distinguishing between components of different radial velocities the use of subscript Arabic numerals is suggested, such as $\mathrm{III}_{1}, \mathrm{III}_{2}, \ldots$; at different times the various components can be identified mainly on the basis of their history. Absorption and emission $\mathrm{V}$ are especially characterized by their connection with the well-known band at $\lambda 4640$. Emissions VI, VII and VIII are sufficiently described by their name.

As far as the spectrum on the whole is concerned, the following classification is proposed:

$Q$ o. This should be the spectrum, at present practically unknown, of a Nova before its outburst. 
Q I. The spectrum before maximum, consisting of absorption I and emission $\mathrm{I}$.

$Q$ 2. The spectrum at maximum, or better, immediately after maximum, consisting of absorption and emission II.

Q 3. The 'diffuse enhanced absorption' stage; a complex spectrum consisting of absorptions and emissions II and III (the latter being often multiple).

Q 4. The 'Orion absorption' stage. The spectrum consists mainly of absorptions and emissions II and IV, but absorption and emission III are also present.

$Q 5$. Two alternative classes, $Q 5 \mathrm{w}$ and $Q 5 \mathrm{f}$; the first is characteristic of fast Novae and the second of slow Novae, although this rule might have exceptions.

Q $5 \mathrm{w}$ consists of emissions and absorptions II, IV and V; sometimes emission and absorption III are faintly present.

$Q 5 \mathrm{f}$ is the 'eta Carinae' stage of slow Novae, characterized by emission VI, but emissions and absorptions II and IV and sometimes III are also present.

$Q 6 \mathrm{w}$ and $\mathrm{Q} 6 \mathrm{f}$ are long transition stages in which emissions $\mathrm{V}$ and VII or respectively VI and VII are present at the same time, with different intensity.

Q 7. The 'nebular stage' consisting practically only of emission VII.

Q 8. The 'Wolf-Rayet' stage. The stellar spectrum consists of emission VIII on a weak continuum; emission VII is also present, but belongs clearly to the nebula.

Q 9. The 'final' stage consisting of emission VII (very faint) for the nebula and a stellar spectrum which in many cases is practically continuous. It cannot be decided at present if spectrum $Q 9$ is identical with spectrum $Q \circ$.

The following items may be added to this general description:

(r) In almost all cases 'transition' stages are defined which often last long; they may be indicated, using two numbers. For instance, $Q x-2$ will be the transition stage from $\mathrm{Q}$ I to $\mathrm{Q} 2$.

(2) In all stages there are peculiarities which cannot be indicated with the preceding short description; especially important is the presence of molecular bands in some Novae.

(3) Attention should be paid to the presence of forbidden lines of O I, N II, etc., in the early stages. A short note may be added in this respect.

(4) In some cases there have been observed 'regressions' from a later stage to an early one, although in a 'normal' Nova the stages as indicated are more or less in chronological order.

(5) The development of some Novae (especially the so-called 'recurrent' Novae like TCrB, RS Oph, T Pyx, etc.) usually does not follow closely the sequence previously described. No suggestions are offered for a classification in these cases.

It is obvious that future work might suggest alterations to the preceding scheme; but it is felt that the present outline may be used advantageously for the preparation of an Atlas of the spectra of Novae. 